\title{
Helical scattering and valleytronics in bilayer graphene
}

\author{
Henning Schomerus \\ Department of Physics, Lancaster University, Lancaster LA1 4YB, United Kingdom
}

(Received 18 August 2010; published 6 October 2010)

\begin{abstract}
We describe an angularly asymmetric interface-scattering mechanism which allows to spatially separate the electrons in the two low-energy valleys of bilayer graphene. The effect occurs at electrostatically defined interfaces separating regions of different pseudospin polarization, and is associated with the helical winding of the pseudospin vector across the interface, which breaks the reflection symmetry in each valley. Electrons are transmitted with a preferred direction of up to $60^{\circ}$ over a large energetic range in one of the valleys, and down to $-60^{\circ}$ in the other. In a Y-junction geometry, this can be used to create and detect valley polarization.
\end{abstract}

DOI: 10.1103/PhysRevB.82.165409

PACS number(s): 72.80.Vp, 73.22.Pr, 85.75.-d

\section{INTRODUCTION}

Research on low-dimensional systems has received a significant stimulus by the recent isolation of atomically thin sheets of graphite, known as graphene. ${ }^{1}$ Graphene's attractive electronic properties can be traced back to a unique low-energy band structure (corresponding to massless Dirac particles in pristine monolayers, which become massive in bilayers), whose key features are dictated by the existence of an intrinsic orbital degree of freedom, known as pseudospin - a choice of two $\pi$ orbitals, presented by the two chemically identical carbon atoms in the unit cell of the graphene honeycomb lattice. ${ }^{2}$ The celebrated phenomenon of chirality ties the pseudospin to the momentum, which results in a Berry phase of $\pi$ in monolayers ( $2 \pi$ in bilayers) whose consequences are observed in the quantum Hall effect ${ }^{3-5}$ and in the chiral Klein tunneling across np junctions. ${ }^{6-8}$

A descended, remarkably robust electronic featureshared by graphene monolayers, bilayers, and multilayers of various stacking order-is the splitting of the low-energy band structure into two energetically degenerate but orbitally inequivalent valleys (of different isospin), situated around the corners of the Brillouin zone (the $\mathrm{K}$ and $\mathrm{K}^{\prime}$ points). In this work we demonstrate that the electrons belonging to the valleys can be separated and detected by a variant of the Klein tunneling setup, which utilizes a certain type of electrostatically defined interfaces in bilayer graphene. The interfaces in question separate regions of opposite pseudospin polarization, and are obtained by combining pairs of oppositely charged top and back gates as illustrated in Fig. 1(a). In the past, such interfaces have attracted attention because they guide valley-dependent states ${ }^{9}$ that propagate along the interface; for transport in the perpendicular direction, they realize a pseudospin variant of a spin valve, ${ }^{10}$ which allows electronic confinement. ${ }^{11}$ By resolving the transport across the interface angularly in each valley, we find that the direction of perfect transmission is skewed away from normal incidence, see Figs. 1(c)-1(h). The broken reflection symmetry is intimately related to a hidden helical structure-the winding of the pseudospin vector as one crosses the interface, which is opposite in the two valleys as illustrated in Fig. 1(b). The ensuing helical scattering favors transmission at opposite angles of incidence and provides a means to separate electrons in the valleys by directing them to different leads.
From a practical perspective, efforts to create an imbalance in the population of the valleys are motivated by the prospects of electronic applications that do not directly rely on the charge of the carriers. ${ }^{12}$ Dubbed valleytronics, this scheme aims to implement analogies to the rapidly maturing field of spintronics, ${ }^{13}$ with the two valleys substituting the two states of physical electronic spin. The original proposal to create and detect valley polarization relies on edge effects; ${ }^{12}$ alternative proposals employ broken sublattice symmetry combined with in-plane electric fields ${ }^{14}$ or drastic doping..$^{15}$ Besides the practical obstacles to implement these routes, these proposals face the severe problem of strong intervalley scattering occurring at pn junctions in monolayer graphene. ${ }^{16}$ In this context, the valley-dependent helical scattering offers two key advantages: The required interfaces can be implemented and controlled by ordinary gates, and intervalley scattering is negligible. Toward the end of this paper, we evaluate the feasibility of this route to valleytronics by numerical results for wide (but finite-sized) Y-junction devices.

The paper is organized as follows: Sec. II presents analytical results for scattering at pseudospin interfaces in bilayer graphene. Section III presents numerical results for valleytronics applications. Section IV contains conclusions. Appendices A and B provide background for the analytical and numerical methods.

\section{HELICAL SCATTERING}

We start our considerations with the scattering at pseudospin interfaces in bulk graphene, which allows a fully analytical treatment that reveals the connection of valleydependent scattering and helical winding.

In bilayer graphene, pseudospin refers to the probability amplitude to find an electron on the top layer [spin up, amplitude $\left.\psi_{\uparrow}(\mathbf{r})\right]$ or on the bottom layer [spin down, amplitude $\left.\psi_{l}(\mathbf{r})\right]$. Pseudospin polarization can be created by oppositely charged top and back gates, which induce an electrostatic potential $U_{\uparrow}=-U_{\downarrow} \equiv U(x)$ in the two layers. Patterning of the gates allows to spatially vary the potential. The relevant lowenergy electronic transport in this landscape can be described by a two-component Hamiltonian ${ }^{17}$ 

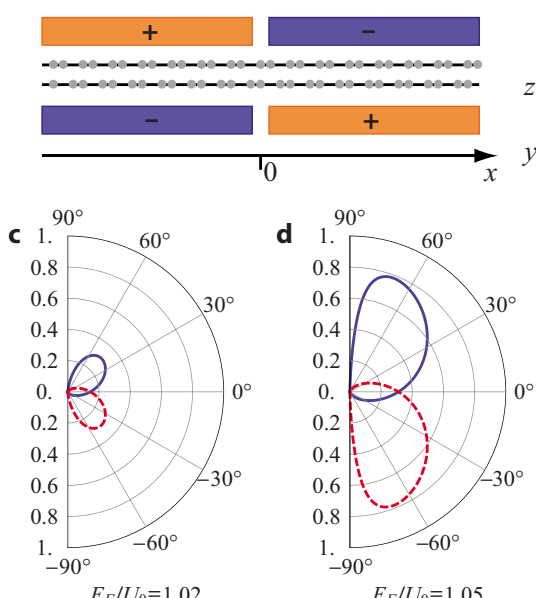

$E_{F} / U_{0}=1.02$

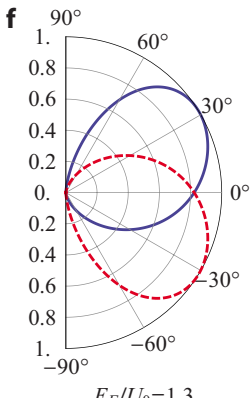

g

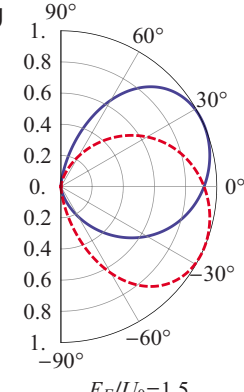

$E_{F} / U_{0}=1.5$

i

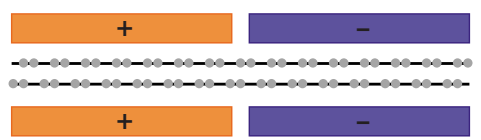

b

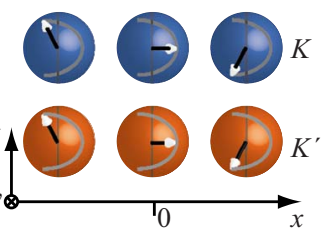

e 1

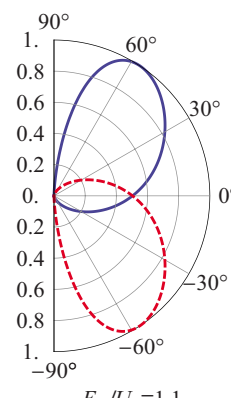

$E_{F} / U_{0}=1.1$

h
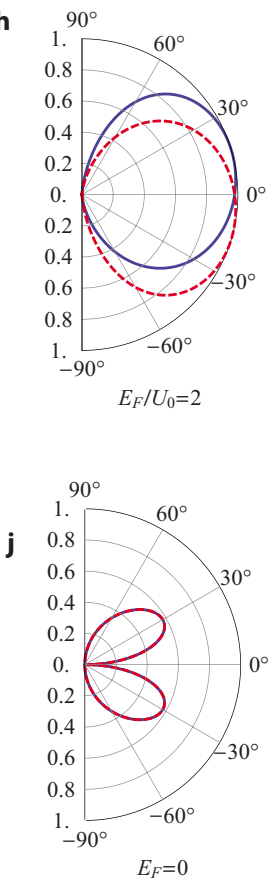

FIG. 1. (Color online) Helical scattering of electrons in bilayer graphene at an interface between regions of different pseudospin polarization. Panel (a) sketches in a side view how such an interface is obtained by sandwiching a bilayer graphene flake between positively and negatively charged gates (light orange and dark blue, respectively; not drawn to scale). Panel (b) shows the rotation of the pseudospin vector in the valleys around the $\mathrm{K}$ and $\mathrm{K}^{\prime}$ points. In the polar plots of panels (c)-(h), solid (blue) lines show the angleresolved transmission probability $T(\varphi)$ in the K valley while dashed (red) lines show this probability in the $\mathrm{K}^{\prime}$ valley. Each panel pertains to a different Fermi energy $E_{F}$, ranging from values just above the gap where the transmission in each valley is angularly asymmetric but small [panels (c) and (d)], over a regime where angular asymmetric scattering occurs around a transparent direction [panels (e)-(g)] to the regime far above the gap, where one approaches valley-independent, angularly symmetric scattering [panel (h)]. Panels (i) and (j) contrast these finding to the case of Klein tunneling at an np junction (Refs. 6 and 7), which does not distinguish the valleys.

$$
H=\left(\begin{array}{cc}
U(x) & -\left(\hat{p}_{x}-i \xi \hat{p}_{y}\right)^{2} / 2 m \\
-\left(\hat{p}_{x}+i \xi \hat{p}_{y}\right)^{2} / 2 m & -U(x)
\end{array}\right),
$$

which acts on the spinor wave function $\psi(\mathbf{r})$ $=\left[\psi_{\uparrow}(\mathbf{r}), \psi_{\downarrow}(\mathbf{r})\right]^{T}$. Here $m$ is the effective mass, and $\hat{p}_{x}$ and $\hat{p}_{y}$ are the two components of the momentum operator in the graphene plane. The valley index $\xi$ takes the values $\xi=1$ in the $\mathrm{K}$ valley and $\xi=-1$ in the $\mathrm{K}^{\prime}$ valley.

A sharp pseudospin interface along the line $x=0$ is described by a steplike profile of the electrostatic potential, with $U(x)=-U_{0}$ for $x<0$ and $U(x)=U_{0}$ for $x>0$. In the regions of constant potential, the electronic bilayer spectrum is gapped so that electrons can only move freely for Fermi energies $E_{F}$ fulfilling $\left|E_{F}\right|>\Delta / 2=\left|U_{0}\right|$. (At energies within the gap, there is a pair of states which propagate along the interface; ${ }^{9}$ these states do not contribute to the transport across the interface. In the following, we will assume $E_{F}$ $>U_{0}>0$.) In each valley, the angle-resolved probability $T(\varphi)=|t(\varphi)|^{2}$ of electrons approaching the interface from the left to be transmitted to the right can be calculated in a standard wave-matching procedure (see Appendix A). The transmission amplitude is

$$
t=2 \frac{i p_{y}^{2}\left(1-\beta^{2}\right) \operatorname{Im} \alpha+i p_{x} q\left[\operatorname{Re} \alpha\left(1+\beta^{2}\right)-\beta\left(1+|\alpha|^{2}\right)\right]}{p_{y}^{2}\left(1-\beta^{2}\right)\left(1-\alpha^{* 2}\right)+i p_{x} q\left[\left(\alpha^{*}-\beta\right)^{2}+\left(1-\alpha^{*} \beta\right)^{2}\right]}
$$

with $\quad p_{y}=p_{0} \sin \varphi, \quad p_{x}=p_{0} \cos \varphi, \quad q=p_{0} \sqrt{1+\cos ^{2} \varphi}, \quad \alpha=$ $-\frac{\left(p_{x}+i \xi p_{y}\right)^{2}}{2 m\left(E_{F}-U_{0}\right)}, \beta=\frac{\left(q-\xi p_{y}\right)^{2}}{2 m\left(E_{F}-U_{0}\right)}$, and $p_{0}=\sqrt{2 m}\left(E_{F}^{2}-U_{0}^{2}\right)^{1 / 4}$. The resulting angle-resolved probability only depends on $E_{F} / U_{0}$, and is plotted in Figs. 1(c)-1(h) as a sequence of polar plots.

Just above the gap $\left(E_{F}=1.02 U_{0}\right)$, the transmission probability in both valleys is small for any angle of incidence (this is the essence of the pseudospin valve effect ${ }^{10}$ ). As the Fermi energy increases, the transmission probability increases but in an angularly asymmetrical fashion: over a large range of energies, electrons in the $\mathrm{K}$ valley have large transmission probability for incident angles in the range of $30^{\circ}$ to $60^{\circ}$, where the interface is almost transparent. The transmission characteristics in the $\mathrm{K}^{\prime}$ valley are similarly skewed but into the opposite direction. An approximately symmetric, valley-independent transmission probability only emerges for energies on the order of $E_{F}=2 U_{0}$ and beyond. These observations can be quantified analytically by considering the direction of unit transmission probability, which exists for values $E_{F} \geq \sqrt{9 / 8} U_{0} \approx 1.06 U_{0}$, and then is given by the momentum vector

$$
\mathbf{p}_{0}=\left(\begin{array}{c}
\sqrt{3 m \sqrt{E_{F}^{2}-U_{0}^{2}}-m E_{F}} \\
\frac{\xi}{2}\left(\sqrt{2 m\left(E_{F}+U_{0}\right)}-\sqrt{2 m\left(E_{F}-U_{0}\right)}\right)
\end{array}\right) .
$$

The component parallel to the interface is finite and has an opposite sign in the two valleys; it only becomes negligible when $E_{F} \gg U_{0}$.

It is natural to inquire whether there is a fundamental mechanism behind this valley-dependent transmission pattern. Here we offer two observations - first, the helical winding of the pseudospin vector for propagating modes as one 
crosses the interface, which manifests the broken reflection symmetry in each valley; second, a condition for the pseudospin vector of evanescent modes, which identifies the transparent direction $\mathbf{p}_{0}$.

The pseudospin vector $\vec{P}=\langle\overrightarrow{\boldsymbol{\sigma}}\rangle$ is a unit vector composed of the expectation values of the Pauli spin matrices. For a constant potential $U$, a propagating wave with momentum $\mathbf{p}$ carries pseudospin

$$
\vec{P}_{\text {prop }}=\frac{p_{y}^{2}-p_{x}^{2}}{2 m E_{F}} \hat{\mathbf{i}}-\frac{\xi p_{x} p_{y}}{m E_{F}} \hat{\mathbf{j}}+\frac{U}{E_{F}} \hat{\mathbf{k}} .
$$

The out-of-plane component is a measure of the pseudospin polarization while the projection onto the graphene plane is linked to the propagation direction (this is the celebrated chirality of charge carriers in graphene ${ }^{2}$ ); notably, the sign of the $y$ component depends on the valley index $\xi$. Far to the left and to the right of the interface, the pseudospin vector has a finite but opposite out-of-plane component. As $U$ changes from $-U_{0}$ to $U_{0}$ the pseudospin vector describes an arc on the unit sphere, which has opposite orientation in the two valleys [see Fig. 1(b)], and thereby explicitly breaks the reflection symmetry in a fixed valley. The asymmetric transmission pattern is therefore connected to a hidden helical structure in each of the two valleys.

Our second observation involves the evanescent modes, which carry pseudospin close to the interface, and whose amplitudes are related to the propagating modes by the wave matching conditions. At the transparency condition [Eq. (3)], the evanescent modes on both sides of the interface have the same amplitude and phase on both layers, $\psi_{\mathrm{ev}} \propto(1,1)^{T}$. Their pseudospin vector $\vec{P}_{\mathrm{ev}}=\hat{\mathbf{i}}$ therefore lies in the graphene plane, and points into the direction perpendicular to the interface. While this condition is the same in both valleys, the properties of the evanescent modes differ; as a result, they meet the condition for the finite, valley-dependent value of $p_{y}=p_{0 y}$ specified in Eq. (3), which again explicitly breaks the reflection symmetry in each valley.

\section{VALLEYTRONICS APPLICATIONS}

We now turn to the prospects to employ helical scattering for valleytronics applications. In view of previous proposals in monolayer graphene, ${ }^{12,14,15}$ a key question is whether pseudospin interfaces induce intervalley scattering in zigzag nanoribbons, as this is the orientation in which the valleys are conserved away from the interface. This question goes beyond the continuum description ${ }^{16}$ and is numerically addressed in Fig. 2. The top panel shows the nanoribbon geometry. To quantify the intervalley scattering, consider the current $I_{1 K}^{l X}$ in lead $l=1,2$ and valley $X=\mathrm{K}, \mathrm{K}^{\prime}$ in response to a bias voltage $V$ applied to lead 1, where the electrons are polarized in valley $\mathrm{K}$ (below we describe how such valley polarization can be created; lead 2 is grounded). For small bias we can define valley- and lead-resolved conductances $G_{1 K}^{I X}=I_{1 K}^{I X} / V$, which can be evaluated using standard quantum transport algorithms (see Appendix B). The bottom panels show valley-resolved transmission coefficients $T_{X}=G_{2 X}^{1 K} / G_{0}$ (where $G_{0}$ is the conductance quantum), as well as reflection
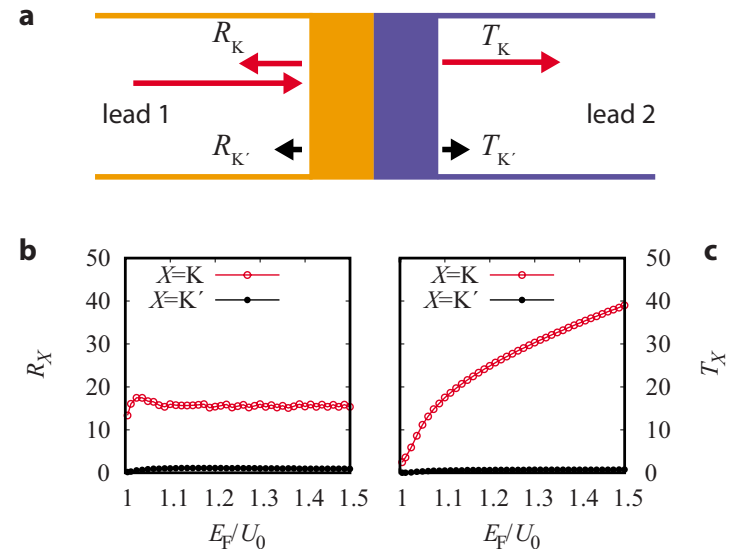

FIG. 2. (Color online) Absence of intervalley scattering. Panel (a): top-view sketch of a (zigzag) graphene nanoribbon geometry with a pseudospin interface, and definitions of reflection and transmission coefficients (summed over all channels) when a $\mathrm{K}$-polarized current is injected into lead 1. Panels (b) and (c): numerical results for the valley-resolved reflection and transmission coefficients (nanoribbon width $W \simeq 150 \hbar / \sqrt{2 m\left|U_{0}\right|}$ ).

coefficients $R_{X}$, defined analogously based on the reflected electron stream, for a wide nanoribbon of width $W$ $\simeq 150 \hbar / \sqrt{2 m\left|U_{0}\right|}$ (for these wide ribbons we do not find any difference between the zigzag and antizigzag edge configurations ${ }^{16}$ ). The results show that intervalley scattering is negligible both in reflection as well as in transmission. Encouragingly, the transmission coefficient exceeds the reflection coefficient once the regime of helical scattering is entered. The results also reveal that for the assumed sharp interface the reflection coefficient is almost energy independent (softening the interface further reduces the reflection and intervalley scattering).

In a suitable geometry, the helical scattering should therefore enable to spatially separate the electrons in the two valleys. To evaluate the feasibility of such a bulk valley filter we consider a Y-junction geometry with three leads, shown in Fig. 3(a), where a pseudospin interface is placed at the entrance of leads 2 and 3. This is done with the intent that electrons injected into lead 1 are preferably transmitted into lead 2 when they are in the $\mathrm{K}$ valley, and into lead 3 when they are in the $\mathrm{K}^{\prime}$ valley. To quantify the anticipated valley polarization, we now consider the current $I_{1}^{l X}$ in lead $l=2,3$ and valley $X=\mathrm{K}, \mathrm{K}^{\prime}$ in response to a bias voltage $V$ applied to lead 1 (the injected current from this lead is now valley unpolarized; leads 2 and 3 are both grounded), and the associated conductances $G_{1}^{l X}=I_{1}^{l X} / V$. Figures 3(b)-3(d) show the energy dependence of these conductances, along with the ratio of the larger over the smaller conductance in each lead. The results demonstrate that a finite valley polarization can indeed be obtained for conditions where the conductance itself is also large $\left(E_{F} \approx 1.1 U_{0}\right.$, where the preferred transmission directions are $\approx \pm 60^{\circ}$ ). By injecting unpolarized currents into leads 2 or 3 , the Y-junction geometry can also be used to create valley polarization in lead 1 ; this is shown in panels (e)-(h). Panels (i)-(l) show how valley polarization in lead 1 is detected by the total current in leads 2 and 3, which is quantified by conductances $G_{1 X}^{l}$ (now $X$ stands for the 

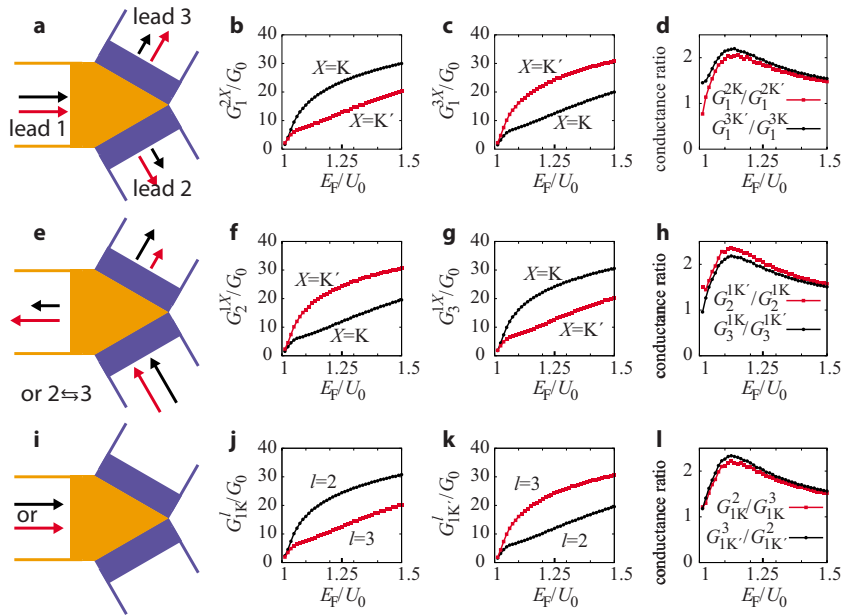

FIG. 3. (Color online) Valleytronics applications. Panel (a): topview sketch of a valley splitter, consisting of a $\mathrm{Y}$ junction where electrons are injected through lead 1, and collected in leads 2 and 3. In this setup, the contact region has the same gate polarity as lead 1, and the incoming current in this lead is valley unpolarized. Panels (b) and (c): valley-resolved conductance of electrons in leads 2 and 3. Panel (d): ratio of the larger to the smaller conductance in these two leads. Analogously, panels (e)-(h) demonstrate that valley polarization can be created in lead 1 by injecting unpolarized currents into lead 2 or 3 . Panels (i)-(1) show how the total current in leads 2 and 3 can be used to the detect the valley polarization of an incoming current in lead 1 .

valley polarization of the incoming current). A $\mathrm{K}$ valleypolarized current injected into lead 1 will preferably divert to lead 2 (panel j) while a $\mathrm{K}^{\prime}$ valley-polarized current will preferably divert to lead 3 (panel k).

Figure 4 shows an alternative design of the $\mathrm{Y}$ junction, where now the contact region has the same gate polarity as leads 2 and 3 . This produces larger conductance ratios for
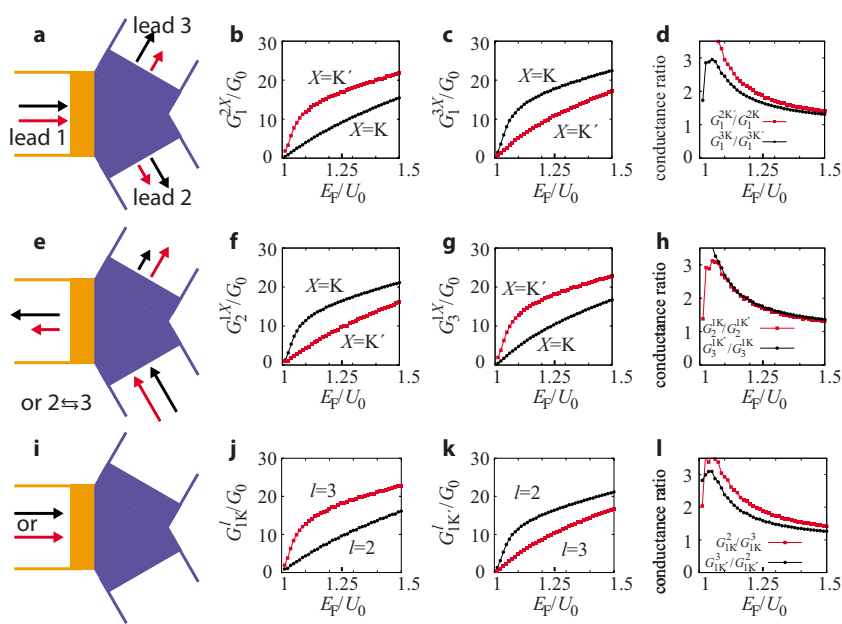

FIG. 4. (Color online) Alternative design of the valley splitters, polarizers, and detectors of Fig. 3, where the contact region has the same gate polarity as leads 2 and 3. Qualitatively, the results for valleys $\mathrm{K}$ and $\mathrm{K}^{\prime}$ are now interchanged; however, the influence of edge states between leads 2 and 3 result in a notable lead asymmetry of the conductance ratios. valley polarization and detection but suffers from larger asymmetries which originate from edge states that connect leads 2 and 3. Symmetry upon interchanging the lead index is restored when one simultaneously inverts the sign of $U_{0}$ and $E_{F}$.

\section{CONCLUSIONS}

In summary, we demonstrated that electrostatically defined pseudospin interfaces in bilayer graphene result in angularly asymmetric, valley-dependent scattering. Above a certain energy threshold, the transmission becomes perfect into a particular direction, which is opposite in the two valleys. This direction only turns toward normal to the interface when energies are very large. The broken reflection symmetry in each valley can be made manifest by considering the helical winding of the pseudospin vector across the interface.

From a conceptual point of view, our findings enforce the role of interfaces to unravel fundamental properties of charge carriers in graphene, thereby complementing the earlier instances of np junctions (which allow to realize Klein tunneling $^{6-8}$ and Veselago lensing $\left.{ }^{18}\right)$, flake boundaries (which can support edge states ${ }^{19}$ ), and regions of large charge carrier density (which induce surface states that support tunneling over large distance ${ }^{20}$ ). From a more practical perspective, the helical scattering opens an avenue to manipulate the valley degree of freedom in bulk graphene.

\section{ACKNOWLEDGMENT}

The author gratefully acknowledges discussions with Elsa Prada, Pablo San-Jose, and Edward McCann.

\section{APPENDIX A: WAVE MATCHING}

The transport problem across the pseudospin interface can be solved by matching the propagating and evanescent modes to the left and right of the interface, which are obtained from the Schrödinger equation $H \psi=E \psi$ with Hamiltonian $H$ given in Eq. (1). The scattering at the interface conserves energy $E$ and the component $p_{y}$ of the momentum parallel to the interface. In the region $x<0$, where $U=-U_{0}$, an incident mode with fixed $p_{y}$ and $E$ has longitudinal momentum $p_{x}=\sqrt{2 m \sqrt{E^{2}-U_{0}^{2}}-p_{y}^{2}} \equiv p$, and is described by a wave function

$$
\psi_{\text {in }}=\exp \left(i p x+i p_{y} y\right)\left(\begin{array}{l}
1 \\
\alpha
\end{array}\right),
$$

where $\alpha=-\frac{\left(p+i \xi p_{y}\right)^{2}}{2 m\left(E-U_{0}\right)}$. The reflected wave

$$
\psi_{\text {ref }}=\exp \left(-i p x+i p_{y} y\right)\left(\begin{array}{c}
1 \\
\alpha^{*}
\end{array}\right)
$$

has longitudinal momentum $p_{x}=-p$. There is also an evanescent mode

$$
\psi_{\text {left }}=\exp \left(q x+i p_{y} y\right)\left(\begin{array}{l}
1 \\
\beta
\end{array}\right),
$$

which decays (to the left, away from the interface) with imaginary momentum $p_{x}=-i q, q=\sqrt{2 m \sqrt{E^{2}-U_{0}^{2}}+p_{y}^{2}}$, and $\beta$ $=\frac{\left(q-\xi p_{y}\right)^{2}}{2 m\left(E-U_{0}\right)}$. 
In the region $x>0$, where $U=U_{0}$, the transmitted wave

$$
\psi_{\text {trans }}=\exp \left(i p x+i p_{y} y\right)\left(\begin{array}{c}
\alpha^{*} \\
1
\end{array}\right)
$$

has longitudinal momentum $p_{x}=p$, and the decaying evanescent mode is given by $p_{x}=i q$,

$$
\psi_{\text {right }}=\exp \left(-q x+i p_{y} y\right)\left(\begin{array}{l}
\beta \\
1
\end{array}\right) .
$$

The wave matching conditions at the interface $x=0$ between the regions are continuity,

$$
\left(\begin{array}{l}
1 \\
\alpha
\end{array}\right)+r\left(\begin{array}{c}
1 \\
\alpha^{*}
\end{array}\right)+c\left(\begin{array}{l}
1 \\
\beta
\end{array}\right)=t\left(\begin{array}{c}
\alpha^{*} \\
1
\end{array}\right)+d\left(\begin{array}{l}
\beta \\
1
\end{array}\right)
$$

and continuity of the first derivative,

$$
p\left(\begin{array}{l}
1 \\
\alpha
\end{array}\right)-p r\left(\begin{array}{c}
1 \\
\alpha^{*}
\end{array}\right)-i q c\left(\begin{array}{l}
1 \\
\beta
\end{array}\right)=p t\left(\begin{array}{c}
\alpha^{*} \\
1
\end{array}\right)+i q d\left(\begin{array}{l}
\beta \\
1
\end{array}\right) .
$$

Together, they form an inhomogeneous system of four linear equations which uniquely determines the four coefficients $r$, $t, c$, and $d$, where, in particular,

$$
t=\frac{2 i p_{y}^{2}\left(1-\beta^{2}\right) \operatorname{Im} \alpha+2 i p q\left[\operatorname{Re} \alpha\left(1+\beta^{2}\right)-\beta\left(1+|\alpha|^{2}\right)\right]}{p_{y}^{2}\left(1-\beta^{2}\right)\left(1-\alpha^{* 2}\right)+i p q\left[\left(\alpha^{*}-\beta\right)^{2}+\left(1-\alpha^{*} \beta\right)^{2}\right]} .
$$

The transmission probability follows from $T=|t|^{2}$ (the corresponding reflection probability is $R=|r|^{2}=1-T$ ). (In the main text, we renamed $E \rightarrow E_{F}$ and $p \rightarrow p_{x}$.) Condition [Eq. (3)], which singles out the propagation direction $\mathbf{p}_{0}$ with unit transmission $T=1$, coincides with the condition $\beta=1$. The transmission amplitude then takes the value $t=(\alpha-1) /(1$ $\left.-\alpha^{*}\right)$.

\section{APPENDIX B: NUMERICAL METHOD}

We base our numerical calculations on a tight-binding model which describes the two low-energy bands of bilayer graphene. This model is obtained from the physical bilayer graphene lattice of carbon atoms, shown in Fig. 5(a), where the $\pi$ orbitals of neighboring atoms in the same layer are coupled by an intralayer hopping energy $\gamma_{0}$ while orbitals of atoms that lie on top of each other (dimer sites) are coupled by the interlayer hopping energy $\gamma_{1}$. At low energies, the latter atoms are only visited in virtual transitions and can be eliminated using decimation. This amounts to solving the
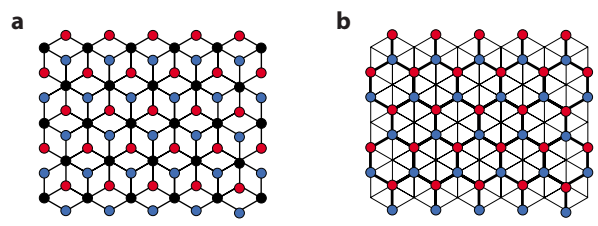

FIG. 5. (Color online) (a) Tight-binding model of a flake of bilayer graphene, consisting of a top and a bottom layer where each layer accommodates a honeycomb lattice of carbon atoms. Black dots denote dimer sites, where carbon atoms of both layers lie on top of each other and are coupled with interlayer coupling constant $\gamma_{1}$. Light (red and blue) dots denote sites of isolated carbon atoms (either on the top or the bottom layer). Lines denote intralayer nearest-neighbor hopping with coupling constant $\gamma_{0}$. (b) Effective model for the two low-energy bands, obtained after decimation (algebraic elimination) of the dimer sites. Thick lines denote coupling with strength $2 \gamma^{\prime}$ while thin lines denote coupling with strength $\gamma^{\prime}$, where $\gamma^{\prime}=\gamma_{0}^{2} / \gamma_{1}$.

Schrödinger equation for the amplitudes on the dimer sites, and inserting the result back into the remaining equations of the nondimer sites. The (generally energy-dependent) selfenergies and mediated hoppings can be set to their values at the Dirac point $E=0$. The resulting two-component tightbinding model is shown in Fig. 5(b). Nearest neighbors are now coupled with strength $2 \gamma^{\prime}$, where $\gamma^{\prime}=\gamma_{0}^{2} / \gamma_{1}$ is an effective hopping energy. Coupling to next-nearest neighbors is still constrained to atoms of different sublattices, and carries strength $\gamma^{\prime}$. This model allows to efficiently enter the regime of many propagating modes in the leads without interfering with the split bands.

The valley-resolved conductances are determined from the relation

$$
G_{1}^{l X}=G_{0} \sum_{\substack{n \in \text { lead } l, \text { valley } X \\ m \in \text { lead } 1}}\left|S_{n m}\right|^{2} .
$$

Here $G_{0}=2 e^{2} / h$ is the conductance quantum, where the factor 2 arises from the degeneracy of the physical spin, and $S_{n m}$ are components of the scattering matrix, which we calculate by a standard numerical decimation method. ${ }^{21}$ The conductances $G_{l}^{1 X}$ and $G_{1 X}^{l}$ are defined and obtained analogously. The valleys are resolved by using the Bloch wave propagation factor $\lambda=\exp \left(i \sqrt{3} a k_{x}\right)$ of modes in leads aligned along the valley-preserving zigzag orientation (here $a$ is the intralayer distance of neighboring carbon atoms). In the $\mathrm{K}$ valley, $\operatorname{Im} \lambda<0$ while in the $\mathrm{K}^{\prime}$ valley $\operatorname{Im} \lambda>0$.
${ }^{1}$ K. S. Novoselov, A. K. Geim, S. V. Morozov, D. Jiang, Y. Zhang, S. V. Dubonos, I. V. Grigorieva, and A. A. Firsov, Science 306, 666 (2004).

${ }^{2}$ A. H. Castro Neto, F. Guinea, N. M. R. Peres, K. S. Novoselov, and A. K. Geim, Rev. Mod. Phys. 81, 109 (2009).

${ }^{3}$ K. S. Novoselov, A. K. Geim, S. V. Morozov, D. Jiang, M. I. Katsnelson, I. V. Grigorieva, S. V. Dubonos, and A. A. Firsov,
Nature (London) 438, 197 (2005).

${ }^{4}$ Yu. B. Zhang, Y. W. Tan, H. L. Stormer, and P. Kim, Nature (London) 438, 201 (2005).

${ }^{5}$ K. S. Novoselov, E. McCann, S. V. Morozov, V. I. Fal'ko, M. I. Katsnelson, U. Zeitler, D. Jiang, F. Schedin, and A. K. Geim, Nat. Phys. 2, 177 (2006).

${ }^{6}$ M. I. Katsnelson, K. S. Novoselov, and A. K. Geim, Nat. Phys. 
2, 620 (2006).

${ }^{7}$ V. V. Cheianov and V. I. Fal'ko, Phys. Rev. B 74, 041403(R) (2006).

${ }^{8}$ A. F. Young and P. Kim, Nat. Phys. 5, 222 (2009).

${ }^{9}$ I. Martin, Ya. M. Blanter, and A. F. Morpurgo, Phys. Rev. Lett. 100, 036804 (2008).

${ }^{10}$ P. San-Jose, E. Prada, E. McCann, and H. Schomerus, Phys. Rev. Lett. 102, 247204 (2009).

${ }^{11}$ L. J. P. Xavier, J. M. Pereira, A. Chaves, G. A. Farias, and F. M. Peeters, Appl. Phys. Lett. 96, 212108 (2010).

${ }^{12}$ A. Rycerz, J. Tworzydło, and C. W. J. Beenakker, Nat. Phys. 3, 172 (2007).

${ }^{13}$ S. A. Wolf, D. D. Awschalom, R. A. Buhrman, J. M. Daughton, S. von Molnár, S. M. L. Roukes, A. Y. Chtchelkanova, and D. M. Treger, Science 294, 1488 (2001).
${ }^{14}$ D. Xiao, W. Yao, and Q. Niu, Phys. Rev. Lett. 99, 236809 (2007).

${ }^{15}$ J. L. Garcia-Pomar, A. Cortijo, and M. Nieto-Vesperinas, Phys. Rev. Lett. 100, 236801 (2008).

${ }^{16}$ A. R. Akhmerov, J. H. Bardarson, A. Rycerz, and C. W. J. Beenakker, Phys. Rev. B 77, 205416 (2008).

${ }^{17}$ E. McCann and V. I. Falko, Phys. Rev. Lett. 96, 086805 (2006).

${ }^{18}$ V. V. Cheianov, V. Fal'ko, and B. L. Altshuler, Science 315, 1252 (2007).

${ }^{19}$ K. Nakada, M. Fujita, G. Dresselhaus, and M. S. Dresselhaus, Phys. Rev. B 54, 17954 (1996).

${ }^{20}$ J. Tworzydło, B. Trauzettel, M. Titov, A. Rycerz, and C. W. J. Beenakker, Phys. Rev. Lett. 96, 246802 (2006).

${ }^{21}$ S. Datta, Electronic Transport in Mesoscopic Systems (Cambridge University Press, Cambridge, UK, 1997). 